A. Pramesh Rao, G. Surarup and Gopal-Krishna, eds.

\title{
Orthogonal Polarization Modes from PSR B0301+19 and B0355+54
}

\author{
R. T. Gangadhara
}

Indian Institute of Astrophysics, Bangalore 5600.34, India

\begin{abstract}
We have analyzed the single pulse data of PSR B0301+19 and $\mathrm{B}(0355+54$ at $1.41 \mathrm{GHz}$ taken from 10()-m Effelsberg radio telescope of MPIfR on 22 and 30 August, 1996. The gray-scale maps are made for the polarization quantities to study the distribution of polarization modes at different pulse longitudes. Circular polarization flips the sign at the pulse center in the case PSR B0355+54 but in the case of PSR $\mathrm{B} 0301+19$ it is mostly positive throughout the pulse. We find the evidence for depolarization that most of the pulsar radiation depolarizes due to the superposition of orthogonal polarization modes.
\end{abstract}

\section{Observation and Data Analysis}

We obtained the high-quality single pulse data of PSR B0301+19 and B0355+54 at $1.41 \mathrm{GHz}$ in August 1996 using the 100-m Effelsberg radiotelescope of MPIfR. The system description and the calibration procedure are given by von Hoensbroech \& Xilouris (1997). For the analysis, we considered about 1000 single pulses for each pulsar. The time resolutions of data of PSR B0301+19 and B0355+54 were $1.35 \mathrm{~ms}$ and $0.05 \mathrm{~ms}$, respectively.

The average polarization parameters: intensity $I$, linear $L$ and circular $V$ polarization are plotted as functions of pulse phase in Figs. 1a and $\mathrm{d}$. The continuous line curve indicates $I$ while the broken and dotted ones represent $L$ and $V$, respectively. The gray-scale maps in Figs. 1b, c, e and $f$ show the frequency of occurrence of orthogonal polarization modes with respect to the pulse phase. The gray-scale maps were made from all those phase bins where the linear polarization $L$ is above $2 \sigma$ level. Here $\sigma$ is the $r m s$ of $L$ in the off pulse region.

\section{Discussion}

The importance of polarization in the pulsar radio emission as a signature of emission mechanism is well known. The leading component in PSR B0301+19 is less linearly polarized compared to the trailing component. While in the case of PSR B0355+54 the leading component is highly polarized compared to the trailing component. The gray-scale maps of polarization angle (Figs. 1b\&e) shows the presence of orthogonal polarization modes and their influence on the linear polarization. When any one of the modes dominate the linear polarization becomes quite high, as indicated by the leading component of PSR B0355+54 

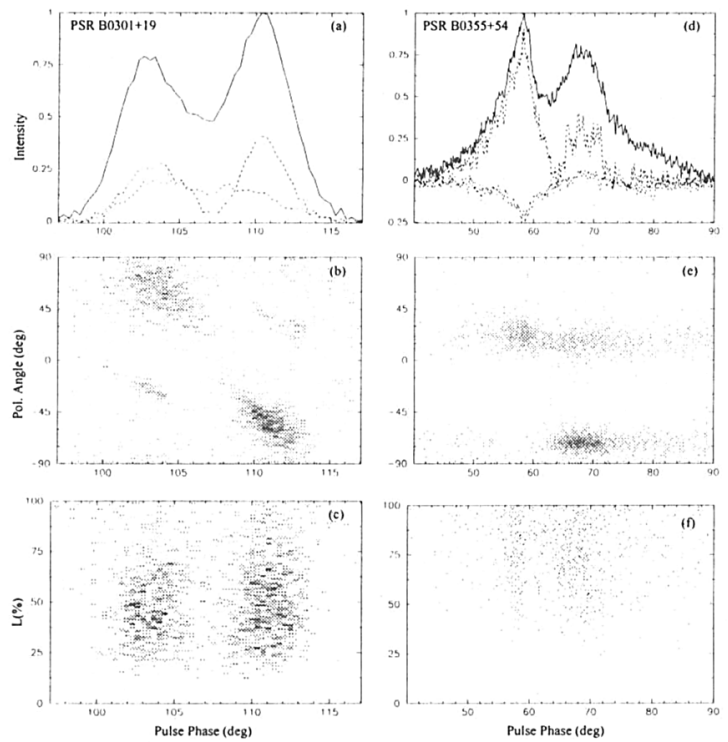

Figure 1. (a)\&(d) are the average profiles of PSR B0301+19 and $\mathrm{B} 0355+54$ at $1.41 \mathrm{GHz}$, while (b)\&(e) are the gray maps of polarization angle obtained from single pulse data, and (c)\&(f) are the percentage of linear polarization gray-scale maps.

(Fig. 1f). When both the modes are present, due to their superposition, linear polarization goes down. The coherent superposition of orthogonal polarization modes can contribute to the non-orthogonal radiation (Gangadhara 1997).

Acknowledgments. I thank R. Wielebinski for providing the data from 100-Effelsberg radiotelescope of MPIfR.

\section{References}

Gangadhara R.T. 1997, A\&A, 327, 155

von Hoensbroech A., Xilouris X.M., 1997, A\&A, 324, 981 